\title{
Stage IVA Mucosal Melanoma of the Head and Neck AJCC v7
}

National Cancer Institute

\section{Source}

National Cancer Institute. Stage IVA Mucosal Melanoma of the Head and Neck A/CC v7. NCI Thesaurus. Code C87533.

Stage IVA includes: (T4a, N0, M0); (T3-T4a, N1, M0). T4a: Moderately advanced disease.

Tumor involves deep soft tissue, cartilage, bone, or overlying skin. T3: Mucosal disease.

NO: No regional lymph node metastases. N1: T umor with regional lymph node metastases. M0: No distant metastasis. (AJCC 7th ed.) 